\title{
La voie de l'AMPc lors de la spermatogenèse: rôle clef du gène CREM
}

La spermatogenèse est un processus de différenciation cellulaire au cours duquel les cellules germinales diploïdes du géniteur se différencient en spermatozoïdes haploïdes. Ce processus est réglé par l'axe hypothalamo-hypophysaire et nécessite l'action coordonnée de plusieurs hormones [1]. En réponse à une stimulation hormonale, les cellules des testicules déclenchent une cascade d'événements qui induisent des changements au niveau du métabolisme cellulaire et de l'expression génique. Les stimulus hormonaux provenant de l'hypophyse sont intégrés au niveau de la membrane cellulaire et transmis au noyau par l'intermédiaire de la voie cytoplasmique de l'AMPc. Cet article sera centré sur l'identification et la fonction des facteurs nucléaires impliqués dans la régulation de l'expression génique par l'AMPc. Nous discuterons du rôle pivot de l'un de ces facteurs, CREM (cAMP-responsive element modulator), au cours du développement des cellules germinales mâles.

Contrôle hormonal dans les testicules : induction de la voie de l'AMPc

La prolifération et la différenciation des cellules germinales dépendent de deux hormones produites par les cellules gonadotropes de l'hypophyse antérieure : la luteinizing hormone (LH) et la follicle-stimulating hormone (FSH) [2, 3]. En l'absence de ces deux hormones, la spermatogenèse se bloque au stade prophase méiotique. Par exemple, la spermatogenèse chez les souris mutantes $h p g$ déficientes en gonatropin releasing hormone est interrompue au stade diplotène nales mâles n'ont pas de récepteurs pour la LH et la FSH, elles reçoivent les signaux hormonaux via les cellules somatiques présentes dans les testicules $[5,6]$. Les récepteurs pour la LH et la FSH sont localisés respectivement sur les cellules somatiques de Leydig et de Sertoli [2, 3]. La stimulation des cellules de Leydig par la $\mathrm{LH}$ conduit à la sécrétion, dans le compartiment interstitiel, de la testostérone qui diffuse dans les tubules séminifères où se trouvent les cellules germinales et les cellules de Sertoli. Après une stimulation par la FSH et par la testostérone, les cellules de Sertoli sécrètent, dans les tubules séminifères, des peptides et d'autres composants nécessaires à la différenciation des cellules germinales [7-9]. La FSH et la LH se lient spécifiquement à leurs récepteurs transmembranaires couplés aux protéines $\mathrm{G}$, ce qui conduit ainsi à la stimulation de l'adénylyl cyclase et à la conversion de l'ATP en AMPc. Le niveau intracellulaire élevé d'AMPc stimule la protéine kinase A (PKA) qui phosphoryle différentes protéines. Parmi les substrats cibles de la PKA on trouve les facteurs de transcription impliqués dans la régulation de l'expression génique. Ces facteurs de transcription se lient à des séquences régulatrices connues pour répondre à l'AMPc (CRE : cAMP responsive element). Ces séquences sont présentes au niveau des régions promotrices de différents gènes. Une fois phosphorylées par la PKA, les protéines se fixant aux sites CRE activent l'expression génique (figure 1) [10].

L'expression des isoformes de la PKA spécifiques des testicules suggère que la voie de l'AMPc dans les testicules possède des fonctions spécialisées qui impliquent la phosphorylation de cibles spécifiques des testicules $(\mathrm{m} / \mathrm{s}$ $n^{\circ} 4$, vol. 9, p. 481) [11-13]. Ici nous discuterons de l'importance de l'une de ces cibles, le facteur de transcription CREM, qui a été récemment identifié dans les testicules et dont le rôle durant la spermatogenèse semble être crucial.

Effecteurs nucléaires de la voie de l'AMPc dans les testicules

Les facteurs nucléaires, dont on sait qu'ils se lient à des séquences CRE, constituent une famille d'au moins dix protéines $[14,15]$. Tous ces facteurs appartiennent à la classe des régulateurs de transcription contenant une leucine zipper et un domaine de fixation à l'ADN [16]. Ces facteurs forment des homo- ou des hétérodimères, suivant un code d'association spécifique. Ainsi, les différentes combinaisons de dimérisation permettent de moduler les possibilités de la régulation génique. L'expression de deux membres de cette famille, CREM et CREB (cAMP-responsive element binding protein), a été décrite dans les testicules $\left(m / s n^{\circ} 4\right.$, vol. 7, p. 506) [17-19]. Ces gènes ont en commun des régions ayant une grande analogie au niveau de leur séquence codante et ont une structure génomique similaire [15]. Le gène CREM, par ailleurs, est remarquable pour plusieurs raisons : (1) son produit contient deux domaines alternatifs de fixation à l'ADN qui sont incorporés individuellement dans la région codante par un épissage alternatif $[20,21]$; (2) contrairement à $C R E B$, qui code seulement pour un activateur, CREM engendre à la fois des activateurs (CREM $\tau$ ) et des ré- 


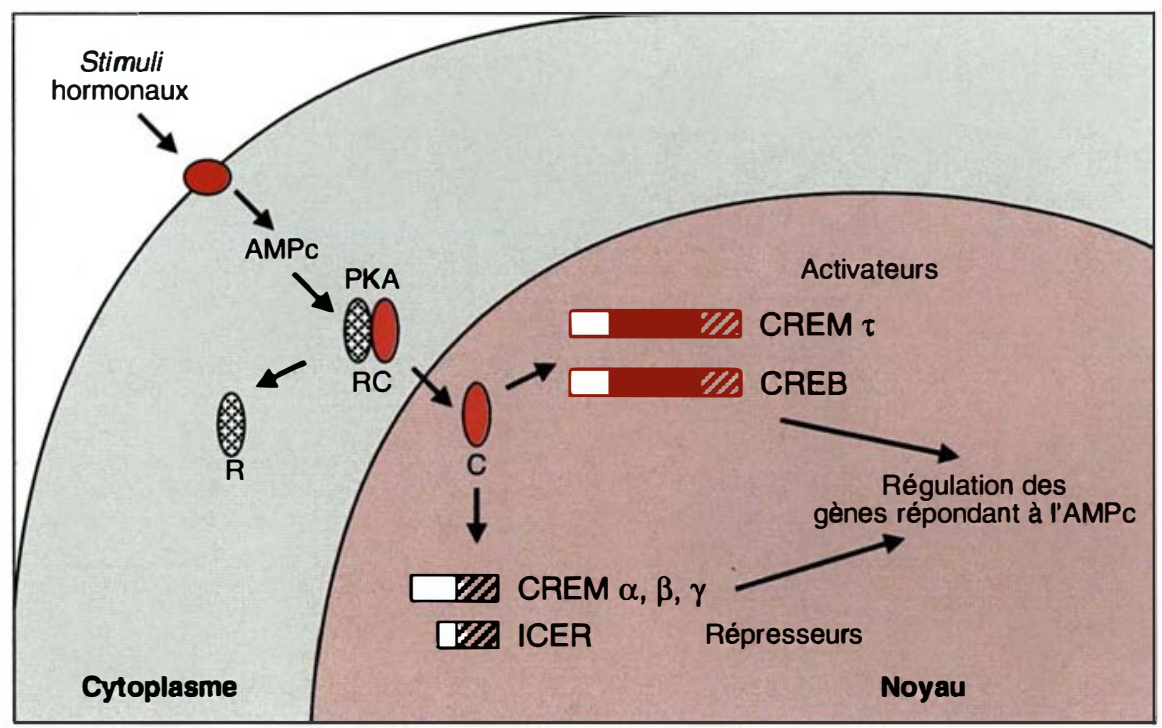

Figure 1. Représentation schématique de la voie de l'AMPc. La fixation de l'hormone sur le récepteur induit une augmentation du niveau intracellulaire $d^{\prime} A M P c$ qui affecte directement l'activité de la protéine kinase $A$ (PKA) [10]. La sous-unité catalytique de la PKA activée (C) est transférée dans le noyau et phosphoryle différentes cibles nucléaires. Parmi les effecteurs de la voie de l'AMPc, on trouve les activateurs CREM $\tau$ et CREB [15]. Ces facteurs appartiennent à la famille des protéines qui, une fois fixées aux sites CRE (cAMP responsive element), règlent l'expression génique. Le gène CREM code pour différentes isoformes dont certaines sont des répresseurs de la transcription induite par l'AMPc (isoformes $\alpha, \beta$ et $\gamma_{i}[15,18,20,21]$ et ICER [24, 25]). Le gène CREB code seulement pour un activateur de la transcrip tion. Les zones rouges représentent les domaines d'activation de la protéine, les zones hachurées les domaines de fixation à l'ADN.

presseurs (CREM $\alpha, \beta, \gamma)$ de transcription, par l'utilisation alternative de domaines riches en glutamines [22] ; (3) CREM présente un profil d'expression spécifique de cellule et de tissu [23, 24]; (4) CREM est un gène à réponse précoce, fortement induit par l'AMPc. Après l'induction, seules des molécules répresseurs (ICER) sont produites par l'utilisation alternative d'un promoteur intronique [24, 25]. Les mécanismes moléculaires de l'activation ou de la répression de la transcription par les protéines CREM ou CREB et la régulation de leur potentiel de transactivation après induction hormonale ont été déjà résumés $[10,15]$.

L'expression de CREM au cours de la spermatogenèse présente des caractéristiques remarquables. CREM est exprimé faiblement avant la méiose et $\mathrm{m} / \mathrm{s} n^{\circ} 4$, vol. 11, avril 95 sation nucléaire de la protéine et sa fixation à l'ADN. La signification physiologique de ces protéines tronquées reste un mystère puiqu'elles ont perdu leur capacité de régler directement l'expression génique. Le rôle de $C R E B$ dans la spermatogenèse reste donc à déterminer.

Chez la souris, la spermatogenèse est un processus cyclique qui a lieu tous les 12 jours. Le cycle de l'épithélium des tubules séminifères a été classé en douze stades représentant les différents types d'associations cellulaires trouvés durant le cycle [26]. L'expression des protéines se liant aux séquences CRE semble dépendre du stade du développement de l'épithélium séminifère. Par exemple, la protéine activatrice issue de CREM est principalement détectée dans les spermatides au stade VII-VIII du cycle alors que le transcrit de $C R E B$ est principalement exprimé dans les cellules de Sertoli au stade III-IV [17, 27]. Ces caractéristiques reflètent une régulation stricte de l'expression génique et suggèrent que ces protéines ne pourraient être fonctionnelles qu'à certains stades de la spermatogenèse. L'épissage alternatif et sa régulation font partie intégrante des mécanismes physiologiques responsables de la différenciation des cellules germinales.

Le gène CREM : régulation et fonction dans les cellules germinales

seules les isoformes répresseurs sont observées. Dans les cellules postméiotiques, un processus d'épissage alternatif provoque un changement fonctionnel de CREM qui se traduit par la commutation des isoformes répresseurs en faveur de l'activateur [18]. Nous avons aussi observé un changement quantitatif dans l'expression de CREM, qui est fortement augmentée à partir du stade pachytène des spermatocytes. En revanche, le gène $C R E B$ est exprimé faiblement à la fois dans les cellules germinales et les cellules somatiques. Toutefois, les cellules somatiques expriment la protéine CREB entière alors que des isoformes tronquées de la protéine CREB sont détectées dans les cellules germinales [17, 19]. Ces isoformes ont des délétions dans leur partie C-terminale, nécessaire pour la locali-
Le changement de l'expression de CREM ayant lieu durant la méiose pose deux questions importantes: (1) Quel est le mécanisme physiologique contrôlant ce changement et celui-ci est-il seulement lié au dévehormonale? (2) Quelle est la fonction de cet abondant activateur CREM dans les cellules germinales?

- La FSH règle l'expression de CREM dans les testicules. Du fait que la spermatogenèse soit contrôlée par des hormones de l'hypophyse, l'importance de la glande hypophysaire dans le contrôle de l'expression de CREM a été étudiée. Des testicules de rats adultes auxquels on a fait subir une ablation de la glande hypophysaire n'expriment pas CREM $\tau$ [28]. loppement ou est-il réglé de façon 
Par ailleurs, chez des animaux prépubères, l'hypophysectomie bloque la commutation dans l'expression de CREM au stade pachytène des spermatocytes. Cela démontre que la glande hypophysaire est directement impliquée dans le maintien et dans le contrôle de la forte expression de CREM $\tau$.

La stimulation hormonale provoquée par la glande hypophysaire a été caractérisée par des études physiologiques sur le hamster [28]. Chez cet animal, les variations saisonnières de l'activité des gonades sont caractérisées par l'arrêt de la spermatogenèse durant l'hiver. Ces changements d'activité dépendent de la photopériode et peuvent être reproduits expérimentalement par une lumière artificielle [29]. Une courte photopériode constante (SP) a pour conséquence une atrophie des gonades en huit semaines [30]. L'atrophie est accompagnée par une réduction de la production de testostérone et d'hormones de la glande hypophysaire $[30,31]$. Le rétablissement d'une longue photopériode (LP) provoque une forte augmentation des niveaux de FSH, de LH et de prolactine, l'augmentation progressive de la taille des testicules et la reprise de la spermatogenèse [30]. Chez les hamsters maintenus constamment en courte photopériode, les testicules restent atrophiés jusqu'à 12 semaines et, après cette période, ils commencent spontanément à se régénérer. L'isof orme CREM $\tau$ ne s'exprime plus dans la gonade atrophiée par l'action de la courte photopériode. Cette expression est restaurée en même temps que l'on rétablit la longue photopériode. L'absence d'expression de CREM $\tau$ dans les gonades atrophiées coïncide avec le faible niveau d'hormones gonadotropes circulantes.

Des injections de FSH à des hamsters maintenus en courte photopériode et ayant donc des testicules complètement atrophiés conduisent à une induction rapide et significative du transcrit CREM $\tau$ [28]. L'expression de CREM $\tau$ chez des animaux ayant reçu des injections de FSH est équivalente à celle des animaux adultes sexuellement actifs.

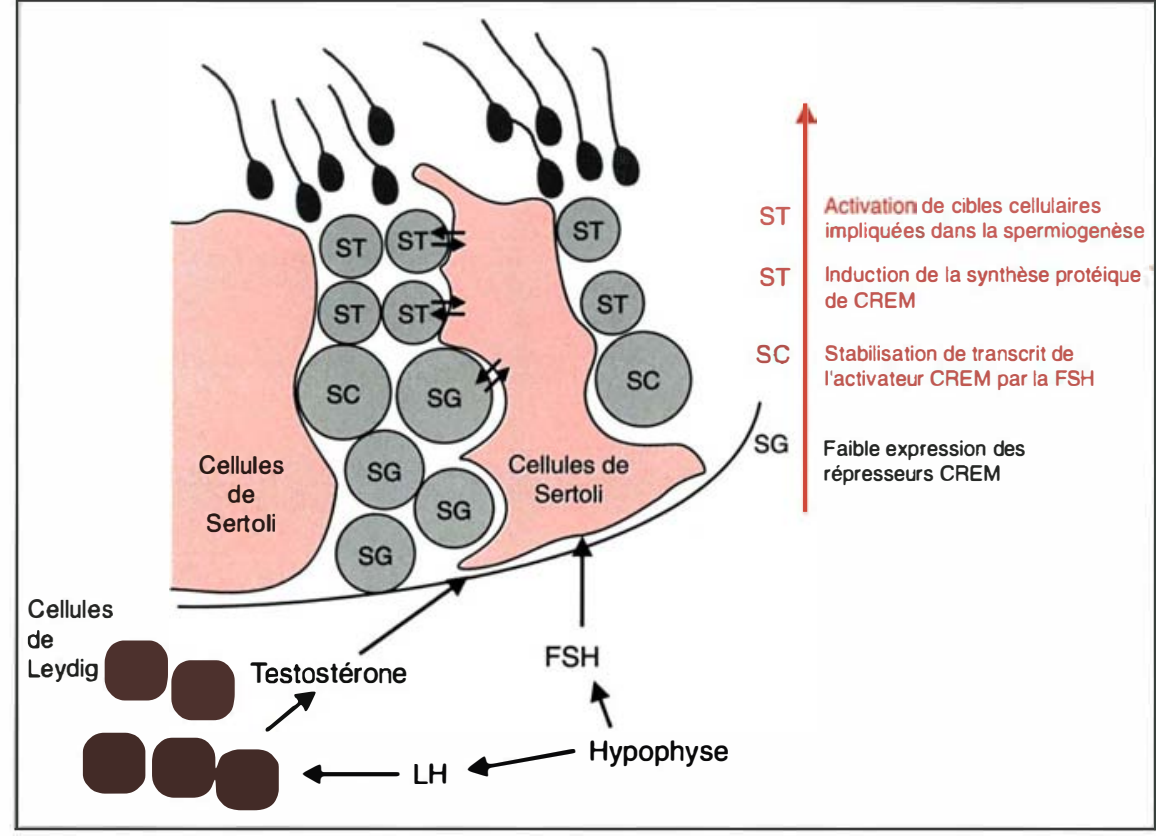

Figure 2. Représentation schématique d'une section de tubule séminifère avec indication du profil d'expression de CREM. L'expression de CREM est réglée à de multiples niveaux durant la spermatogenèse. Les cellules germinales préméiotiques (spermatogonies, SG) expriment faiblement les isoformes répresseurs de CREM. Durant la prophase méiotique, la follicle stimulating hormone (FSH) hypophysaire est responsable de la stabilisation des transcrits de l'activateur CREM dans les spermatocytes (SC) ; la protéine CREM est détectée seulement après la méiose dans les spermatides haploïdes (ST). II est à noter les relations étroites entre les cellules de Sertoli et les cellules germinales (flèches). Dans les spermatides haploïdes, les protéines CREM activent un certain nombre de gènes cellulaires exprimés spécifiquement durant la maturation des spermatides [27].

l'induction du transcrit de l'activateur CREM est due à l'utilisation alternative d'un site de polyadénylation, au niveau du transcrit de CREM, qui supprime la région 3' non traduite. Cela a pour conséquence d'exclure des éléments déstabilisateurs et de rendre le transcrit plus stable [28]. Ces résultats montrent les effets d'une stimulation hormonale sur la stabilité du messager d'un facteur de transcription. Ils démontrent aussi que la FSH règle la fonction de facteurs nucléaires de la voie de l'AMPc, dans les cellules germinales.

- CREM, un régulateur de l'expression génique dans les cellules germinales haplö̈des. La première indication du rôle de CREM durant la spermatogenèse a été donnée par son profil d'expression protéique. Dans l'épithélium séminifère, les transcrits de CREM s'accumulent dans les spermatocytes et les spermatides mais, en revanche, la protéine CREM est détectée seulement dans les spermatides [27]. La fonction de CREM est donc restreinte à un type spécifique de cellules germinales, les spermatides haploïdes. L'absence de la protéine CREM dans les spermatocytes reflète un contrôle strict de la traduction. Il sera important d'analyser ultérieurement le mécanisme de cette traduction retardée et de définir si celle-ci est sous un contrôle hormonal. La phosphorylation par la PKA active la fonction de CREM, permettant le relais du signal hormonal 
du cytoplasme vers le noyau [32]. L'activateur CREM est efficacement phosphorylé par la PKA dont l'activité endogène dans les spermatides est dépendante de l'AMPc, indiquant que la protéine CREM est une cible nucléaire de la voie de l'AMPc dans les cellules spermatogéniques haploïdes [27].

La détection de la protéine activatrice CREM dans les spermatides coïncide avec l'activation transcriptionnelle de plusieurs gènes contenant un motif CRE dans leur promoteur. Ces gènes codent principalement pour des protéines de structure nécessaires à l'assemblage des spermatozoïdes (protéines de transition, protamine RT7, etc.), suggérant un rôle pour CREM dans l'activation des gènes nécessaires à la phase tardive de différenciation des spermatides. Cette observation implique que la transcription des gènes structuraux est directement liée à un contrôle hormonal qui permet une modulation du niveau de l'AMPc dans l'épithélium séminifère. Une preuve du rôle de CREM dans l'expression de l'un de ces gènes, $R T 7$, a été apportée lors d'expériences de transcription in vitro. Un anticorps spécifique de CREM bloque la transcription in vitro de $R T 7$ dans des extraits nucléaires des tubules séminifères mais pas dans des extraits de foie [27]. En conclusion, au cours du développement, CREM devrait participer dans les testicules à la régulation de gènes contenant un site CRE dans leur promoteur, en exprimant faiblement les isoformes répresseurs avant la méiose et fortement les isoformes activateurs après la méiose.

\section{Conclusion}

Il est connu depuis plusieurs années que les cellules de Sertoli, par l'intermédiaire de contacts étroits, transmettent la stimulation hormonale aux cellules germinales en maturation. L'analyse des cibles nucléaires de la voie de l'AMPc fournit des informations importantes sur les mécanismes moléculaires par lesquels le signal hormonal est transmis au noyau. La figure 2 résume les événements successifs expliquant comment la stimulation par la FSH module le profil de l'expression génique dans les cellules germinales. Des modèles animaux, ayant des mutations dans les gènes codant pour les protéines se liant aux sites CRE, devraient permettre d'éclairer davantage le rôle de ces facteurs de transcription et d'identifier leurs cibles spécifiques in vivo. A ce propos, le phénotype de la souris chez laquelle le gène $C R E B$ a été détruit par recombinaison homologue [33] suggère une redondance dans la réponse nucléaire à l'AMPc. En revanche, du fait de son importance physiologique dans la différenciation des cellules germinales, il est probable que la destruction du gène CREM aurait des conséquences significatives sur la spermatogenèse

Les recherches conduites au laboratoire de Paolo Sassone-Corsi à Strasbourg, France, sont effectuées par: Nicholas S. Foulkes, Florence Schlotter, Denis Masquilier, Francois Nantel, Monica Lamas, Barbara Bardoni, Enzo Lalli, Janet S. Lee et Lucia Monaco.

\section{Denis Masquilier \\ Nicholas S. Foulkes \\ Florence Schlotter \\ Lucia Monaco \\ Paolo Sassone-Corsi}

Laboratoire de génétique moléculaire des eucaryotes, Cnrs-Inserm U. 184 , Faculté de médecine, 11, rue Humann, 67085 Strasbourg, France.

\section{TIRÉS À PART}

P. Sassone-Corsi.

\section{RÉFÉRENCES}

1. Veldhuis JD. The hypothalamic-pituitarytesticular axis. In : Yen SSC, Jaffe RB, eds. Reproductive endocrinology. Philadelphia: WB Saunders Company, 1991 : 409-59.

2. Steinberger E. Hormonal control of mammalian spermatogenesis. Physiol Rev $1971 ; 51: 1-22$

3. Lostroh AJ. Hormonal control of spermatogenesis. In : Spilman CH, Lobl TJ, Kirton KT, eds. Regulation mechanisms of male reproductive physiology. Amsterdam : Excerpta Medica, $1976: 13-23$.

4. Cattanach BM, Iddon CA, Charlton HM Chiappa SA, Fink G. Gonadotrophin releasing hormone deficiency in a mutant mouse with hypogonadism. Nature 1977; 269 : 33840.

5. Moore RY. Neuroendocrine regulation of reproduction. In : Yen SSC, Jaffe RB, eds. Reproductive endocrinology. Philadelphia: Saunders Company 1978 : 335-78.

6. Santen RJ. The testis. In : Felig P, Baxter JD, Broadus AE, Frohman LA, eds. Endocrinology and metabolism. New York: McGraw Hill Co, 1987 : 821-905.

7. Russell LD. Sertoli-germ cell interrelations: a review. Gamete Res $1980 ; 3$ : 179-202.

8. Grootegoed JA, Oonk RB, Toebosch AMW, Jansen R. Extracellular factors that contribute to the development of spermatogenic cells. In: Stefanini M, Conti M, Geremia R, Ziparo E, eds. Molecular and cellular endocrinology of the testis. Amsterdam: Excerpta Medica, 1986 : 215-225.

9. Jégou B, Syed V, Sourdaine P, Byers S, Gérard N, Velez de la Calle J, Pineau C, Garnier DH, Bauché F. The dialogue between late spermatids and Sertoli cells in vertebrates: a century of research. In : Nieschlag E, Habenicht UF, eds. Spermatogenesis. Fertilization. Contraception. Molecular, cellular and endocrine events in male reproduction. Schering Foundation. New York: SpringerVerlag, 1992 : 56-95.

10. Lalli E, Sassone-Corsi P. Signal transduction and gene regulation: the nuclear response to cAMP. I Biol Chem 1994 ; 269 : 17359-62.

11. Pariset C, Feinberg J, Dacheux JL, Oyen $O$, Jahnsen T, Weinman S. Differential expression and subcellular localization for subunits of cAMP-dependent protein kinase during ram spermatogenesis. J Cell Biol 1989 ; $109: 1195-205$.

12. Oyen O, Scott JD, Cadd GG, McKnight GS, Krebs EB, Hansson V, Jahnsen T. A unique mRNA species for a regulatory subunit of cAMP-dependent protein kinase is specifically induced in haploid germ cells FEBS Lett 1988 ; 229 : 391-4.

13. Lonnerberg P, Parvinen M, Jahnsen T Hansson V, Persson H. Stage-and cell-specific expression of cyclic adenosine 3,5 -monophosphate-dependent protein kinases in rat seminiferous epithelium. Biol Reprod $1992 ; 46: 1057-68$ 
14. Habener J. Cyclic AMP response element binding proteins: a cornucopia of transcription factors. Mol Endocrinol 1990 ; 4:1087-94.

15. de Groot RP, Sassone-Corsi P. Hormo nal control of gene expression: multiplicity and versatility of cyclic adenosine 3', 5'-monophosphate-responsive nuclear regulators. Mol Endocrinol 1993 ; 7 : 145-53.

16. Busch SJ, Sassone-Corsi P. Dimers, leucine zippers and DNA binding domains Trends Genet $1990 ; 6: 36-40$.

17. Waeber G, Meyer TE, LeSieur M, Hermann HL, Gérard, N, Habener J. Developmental stage-specific expression of cyclic adenosine 3, 5' monophosphate response element-binding protein CREB during spermatogenesis involves alternative exon splicing. Mol Endocrinol 1991 ; 5 : 1418-30.

18. Foulkes NS, Mellstrōm B, Benusiglio E, Sassone-Corsi P. Developmental switch of CREM function during spermatogenesis from antagonist to transcriptional activator Nature $1992 ; 355: 80-4$

19. Ruppert S, Cole TJ, Boshart M, Schmid $M$, Schutz G. Multiple mRNA isoforms of the transcription activator protein CREB generation by alternative splicing and specific expression in primary spermatocytes. EMBO J 1992 ; 11 : 1503-12.

20. Foulkes NS, Borrelli E, Sassone-Corsi P. CREM gene: use of alternative DNA binding domains generates multiple antagonists of cAMP-induced transcription. Cell $1991 ; 64: 739-49$.

21. Laoide BM, Foulkes NS, Schlotter F, Sassone-Corsi $\mathbf{P}$. The functional versatility of CREM is determined by its modular structure. $E M B O J$ J 1993 ; 12 : 1179-91.

22. Foulkes NS, Sassone-Corsi P. More is better: activators and repressors from the same gene. Cell $1992 ; 68: 4114$

23. Mellstrōm B, Naranjo JR, Foulkes NS Lafarga M, Sassone-Corsi P. Transcriptional response to cAMP in brain: specific ditribution and induction of CREM antagonists. Neuron $1993 ; 10: 655-65$.

24. Stehle JH, Foulkes NS, Molina CA, Simonneaux V, Pévet $P$, Sassone-Corsi $P$.
Adrenergic signals direct rythmic expression of transcriptional repressor CREM in the pineal gland. Nature $1993 ; 365: 314-20$.

25. Molina CA, Foulkes NS, Lalli E, SassoneCorsi P. Inducibility and negative autoregulation of CREM: an alternative promoter directs the expression of ICER, an early response repressor. Cell $1993 ; 75: 875-86$.

26. Oakberg J. A description of spermiogenesis in the mouse and its use in analysis of the cycle of the seminiferous epithelium and germ cell renewal. Am J Anat 1956; 99 : 504-16.

27. Delmas V, van der Hoorn F, Mellström B, Jégou B, Sassone-Corsi P. Induction of CREM activator proteins in spermatids: downstream targets and implications for haploid germ cell differentiation. Mol Endocrinol $1993 ; 7: 1502-13$.

28. Foulkes NS, Schlotter F, Pévet P, Sasso ne-Corsi P. Pituitary hormone FSH directs the CREM functional switch during spermatogenesis. Nature $1993 ; 362: 264-7$.

29. Desjardins C, Ewing LL, Johnson BH. Effects of light deprivation upon the spermatogenic and steroidogenic elements of hamster testes. Endocrinology 1971 ; 89 : 791 -

30. Crowley WF Jr, Whitcomb RW, Jameson JL, Weiss J, Finkelstein JS, O'Dea LS. Neuroendocrine control of human reproduction in the male. Rec Progr Horm Res 1991 ; $47: 27-43$.

31. Jetton AE, Fallest PC, Dahl KD, Schwartz NB, Turek FW. Photoperiodic differences in vitro: gonadotropin basal secretion and gonadotropin-releasing hormone responsiveness in the golden hamster. Endocrnology 1991 ; 129 : 1025-31.

32. de Groot RP, den Hertog I, Vandenheede JR, Goris J, Sassone-Corsi P. Multiple and cooperative phosphorylation events regulate the CREM activator function. EMBO J 1993 ; 12 : 3903-11.

33. Hummler E, Cole TJ, Blendy JA, Ganss $R$, Aguzzi A, Schmid $W$, Beermann $F$ Schütz G. Targeted mutation of the CREB gene: compensation within the CREB/ATF family of transcription factors. Proc Natl Acad Sci USA $1994 ; 91$ : 5647-51. 